\title{
Transdiagnostic treatment of emotional disorders in people with multiple sclerosis: randomized controlled trial
}

\author{
Nabi Nazari* (D, Masood Sadeghi, Ezatolah Ghadampour and Davod Mirzaeefar
}

\begin{abstract}
Background: Multiple sclerosis (MS) is a neurodegenerative disease of the central nervous system. MS is significantly associated with a high rate of psychological, behavioral, and emotional consequences. Despite the frequent mental disorders, high rate of psychological comorbidities, and emotional problems in people with MS (PwMS), these conditions are often underdiagnosed and undertreated. This study aimed to examine the efficacy of a group format of the unified protocol for the transdiagnostic treatment of emotional disorders in adult PwMS associated with an emotional disorder.
\end{abstract}

Methods: Seventy adult PwMS were randomized using an internet-based computer system to either the unified protocol $(n=35)$ or treatment as usual condition. The assessment protocol included semi-structured clinical interviews and self-reports evaluating diagnostic criteria, depression, anxiety and worry symptoms, emotional dysregulation, and affectivity.

Results: The parametric test of analysis of covariance, followed the intent to treat analyses, revealed the unified protocol significantly changed depression symptoms (Cohen's $d=1.9$ ), anxiety symptoms (Cohen's $d=2.16$ ), worry symptoms (Cohen's $d=1.27$ ), emotion dysregulation (Cohen's $d=0.44$ ), positive affect (Cohen's $d=1.51$ ), and negative affect (Cohen's $d=1.89$ ) compared with the control group. The unified protocol also significantly improved outcome scores at the end of treatment relative to baseline $(p<.001)$.

Conclusion: The findings support that the unified protocol could be an additional efficient psychological treatment for PwMS.

Trial registration IRCT, number: IRCT20190711044173N1. Registered 31 october 2019, https://en.irct.ir/user/trial/40779/ view.

Keywords: Unified protocol, Emotion regulation, Transdiagnostic, Depression, Anxiety trial registration authority

\section{Background}

Multiple sclerosis (MS) is a chronic, progressive, neurodegenerative disease of the central nervous system. MS is significantly associated with psychological, behavioral, and emotional consequences [1]. For people with MS (PwMS), the risk of being affected by an emotional

*Correspondence: Nazariirani@gmail.com

Department of Psychology, Faculty of Human Sciences, Lorestan

University, Khorramabad, Iran disorder (such as depression, anxiety, anger, euphoria) is higher than healthy populations and other chronic conditions [2]. Depression, experienced by up to $50 \%$ of PwMS, can negatively impact functioning, disability, pharmacological therapy adherence, and suicidal ideation [3, 4]. In addition to specific-disorder, psychological comorbidity is common in PWMS and is correlated with a greater disability over time [5]. Suicidal behaviors in PwMS are two times higher than the general population [6]. Besides depression, maladaptive coping strategy 
and emotional dysregulation were the most potent predictors that have predictive accuracy for suicidal ideation as many as $85 \%$ [7]. This neurological disease that affects the limbic system will induce emotional disturbances. Also, comorbidity has an additive adverse effect on patients' mental quality of life and is associated with an increased risk of debilitating complications, further increasing disease burden. For instance, during the MS, risk-related behaviors may expose an individual to various problematic environmental agents [8]. Despite the frequent mental disorders, comorbidities, and emotional problems in PwMS, these conditions are often underdiagnosed and undertreated [9].

Several reasons for this underdiagnosed condition have been documented. In a neurologic setting, evidence highlights the weakness of the DSM criteria application [10]. Also, the MS syndrome's heterogeneous nature and the potential for confusing specific somatic complaints of MS (e.g., fatigue) with depression symptoms may lead to falsely elevated underdiagnoses rates. Moreover, disorder-specific interventions and treatments based on primary and secondary diagnoses are not suggested to be effective with complex cases [11]. Furthermore, Disorder-specific protocols can be difficult to justify when the clinical reality is complex, and comorbidities are the norm, particularly in chronic somatic disease (e.g., MS) [12].

Cognitive Behavioral Therapy (CBT) programs have demonstrated effectiveness in promoting mental health in PwMS for treating depression [13]. Nevertheless, effective treatments for anxiety are lacking [14]. Recent findings have shown that CBT was less efficient than other interventions in the psychological treatment of PwMS [15]. Transdiagnostic and integrated therapies have emerged as recommended approaches for the treatment of several co-occurring mental health disorders, as they provide a more parsimonious [16] and more efficient strategy to working with comorbid presentations [17]. Some studies have suggested that a transdiagnostic treatment approach for PwMS can be appropriate [18].

Transdiagnostic approaches refer to identifying the etiology and maintenance mechanisms that are common in multiple disorders [19]. In emotional disorders, neuroticism has been considered a key etiology mechanism shared by all emotional disorders [20]. Other mechanisms identified have been rumination, suppression, anxiety sensitivity, and misappraisal [21], frequently reported in PwMS [22]. These mechanisms can increase or maintain persistent negative emotions and may affect physical and psychological functioning. From this perspective, transdiagnostic treatment consists of techniques that serve to target an identified set of underlying core processes [19]. Emotion regulation seems to play a critical role in the treatment of complex cases, diagnoses with a combination of psychological risk factors, or comorbidities [23]. PwMS experience higher rates of negative emotions related to different situations such as support family members, body image, pregnancy worry, uncertainty about the relationship, and sexual dysfunction [24, 25].

The Unified Protocol is a CBT transdiagnostic emotion-focused skill-based therapy [26, 27]. The unified protocol has been manualized to be applied to the treatment of anxiety disorders, depression, and other emotional disorders in which emotion dysregulation is a core component [28]. The protocol has been adopted in 12 to 14 sessions in a group format [29]. Numerous studies have supported the efficacy of the unified protocol in improvements on anxiety and depression symptoms, functional impairment, and well-being $[27,30]$ chronic diseases [31], and social, job, and general performance [32].

\section{Current study}

The unified protocol is equally effective as gold-standard specific disorder protocols for individuals with comorbid emotional disorders [27]. Regarding the prevalence of emotional disorders, high comorbidities, frequent emotional problems, and the high prevalence of the riskybehaviors during the MS [8], the application of unified protocol, as an emotion-focused, skill-based intervention, could be beneficial through targeting emotion regulation mechanism, improvement emotional disorder comorbid conditions, and temperamental changes concerning neuroticism. However, there is a lack of empirical evidence on the unified protocol and MS. The purpose of the study was to examine and develop the efficacy of a group format of the unified protocol for adult PwMS with depression or anxiety symptoms. We hypothesized that at post-intervention, treatment group participants would show significant improvements in measure of depression, measures of anxiety and worry, the measure of emotion regulation, and the measure of affectivity relative to the treatment-as-usual (TAU) group. Also, we hypothesized that treatment group participants would demonstrate significantly improve on dependent variable scores compared with baseline at post-intervention.

\section{Methods \\ Participants}

The consort diagram is illustrated in Fig. 1. Participant recruitment efforts included notifying MS clinics and MS associations through the use of brochures and posters. A total of 122 people were assessed to participate in the trial. Of these, 70 (44 females) met all eligibility criteria. Participants' ages ranged from 22 to 47 years. The mean age of the participants was $35.30(S D=3.01)$. The 


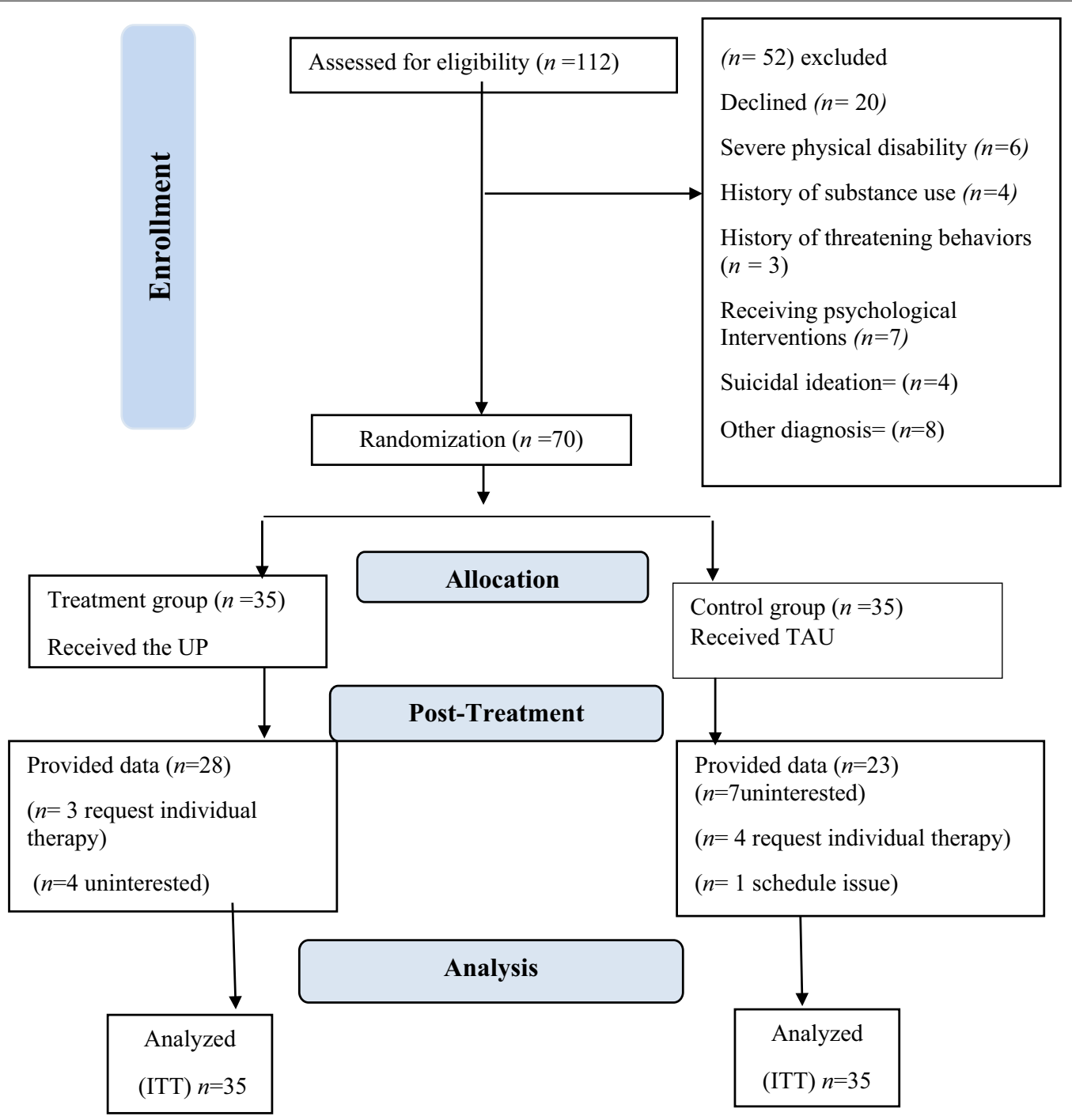

Fig. 1 The participants flow chart diagram. UP unified protocol, TAU treatment as usual, ITT intent to treat, $n$ frequency

participants were generally well-educated. The baseline assessment is demonstrated in Table 1.

Inclusion criteria included (a) fluent in Persian, (b) at least 18 years of age, (c) a valid diagnosis of MS, (d) obtained a consort, (e) received a diagnosis of depression or anxiety disorders based on DSM-IV [33] [Diagnostic and Statistical Manual of Mental Disorders, fourth edition; American Psychiatric Association, 2000], (f) medical agreement or valid referral document for participation.

Exclusion criteria included (a) present or history diagnosis of schizophrenia, psychosis, or organic mental disorder, (b) other chronic physical illnesses, (c) pregnancy or Breast-feeding, (d) risk or history of threatening behaviors, (e) missed three consecutive sessions (f) receiving psychological or psychiatric treatments during the study (e.g., antidepressant or anxiolytic medication), (g) moderate to high cognitive impairment or physical disabilities.

\section{Measures}

Structured Clinical Interview for DSM-IV Axis I Disorders [SCID I-IV: 34, 35] was used in the current study. The diagnosis was moderate to good (Kappa coefficient higher than (0.6). Most interviewees and interviewers reported the desirable implementation of the local version of SCID-I. Kappa was higher than 0.4 for all the diagnoses except for Generalized Anxiety Disorders. The Kappa was above 0.85 in most of the diagnoses, and in half, it was above 0.9 , indicating acceptable reliability 


\begin{tabular}{|c|c|c|c|}
\hline Item Characteristic & Value & Test & $p$ \\
\hline \multicolumn{4}{|l|}{ MS duration, $n(\%)$} \\
\hline to 3 years & $39(28.2)$ & $x^{2}=0.92$ & .396 \\
\hline 3 to 7 years & $31(44.75)$ & & \\
\hline \multicolumn{4}{|l|}{ Gender, $n(\%)$} \\
\hline Women & $43(63.4)$ & $x^{2}=3.65$ & .056 \\
\hline Man & $27(38.6)$ & & \\
\hline \multicolumn{4}{|l|}{ Education, $n(\%)$} \\
\hline Primary education & $12(17.1)$ & $x^{2}=30.22$ & $<.001$ \\
\hline Higher education & $58(82.9)$ & & \\
\hline \multicolumn{4}{|l|}{ Marital, n (\%) } \\
\hline Single & $28(40)$ & $x^{2}=2.80$ & .091 \\
\hline In relationship & $42(60)$ & & \\
\hline \multicolumn{4}{|l|}{ Principal diagnosis SCID } \\
\hline \multicolumn{4}{|c|}{ Depressive disorder, $n(\%)$} \\
\hline MDD & $34(48.6)$ & & \\
\hline Dysthymia & $5(7.1)$ & & \\
\hline \multicolumn{4}{|l|}{ Anxiety disorder, $n(\%)$} \\
\hline GAD & $22(31.4)$ & & \\
\hline SAD & $9(12.9)$ & & \\
\hline \multicolumn{4}{|l|}{ Continues variables } \\
\hline Age, $M(S D), y$ & $35.13(5.28)$ & $t(68)=1.01$ & .321 \\
\hline MS duration, $M(S D), y$ & $3.31(1.37)$ & $t(68)=1.03$ & .304 \\
\hline PANAS-PA, $M((\mathrm{SD})$ & $26.00(3.75)$ & $t(68)=1.55$ & .122 \\
\hline PANAS-NA, M ((SD) & $27.42(2.72)$ & $t(68)=-0.6$ & .148 \\
\hline PSWQ, M (SD) & $47.55(9.46)$ & $t(68)=-1.23$ & .221 \\
\hline HADS-A, M (SD) & $12.43(1.37)$ & $t(68)=-1.89$ & .059 \\
\hline HADS-D, M ((SD) & $12.84(1.63)$ & $t(68)=-1.24$ & .214 \\
\hline DERS, $M((\mathrm{SD})$ & $110.14(12.17)$ & $t(68)=-1.50$ & .132 \\
\hline
\end{tabular}

$n$ frequency, $y$ years, SCID-I-IV structured clinical interview for DSM-IV axis i disorders, MDD major depressive disorder, GAD generalized anxiety disorder, $S A D$ social anxiety disorder, $t$ independent $t$ test, $S D$ standard deviation, PANAS-PA positive and negative affect schedule-positive affect, PANAS-NA positive and negative affect schedule-negative affect, HADS-A the hospital anxiety and depression scale-Anxiety, HADS-D the hospital anxiety and depression scale-depression, PSWQ Penn State Worry Questionnaire, DERS difficulties in emotion regulation scale

[36]. (Note: at the time of the study, a Persian version of the SCID for DSM-V was not yet validated. The clinical psychologists and supervisors on the study created a cross-referenced checklist and determined that patients met criteria for anxiety or depression based on both the DSM-IV and DSM-V.)

\section{Primary outcomes measures}

The hospital anxiety and depression scale [HADS: 37]. The HADS is a highly reliable screening measure for assessing anxiety and depression in PwMS. The HADS consists of 14-items, two sub-scales, 7-items for anxiety (HADS-A), and seven items for depression (HADS-D).
A suggested cutoff score of 11 demonstrated high sensitivity (90\%) and specificity (92\%) for the Anxiety subscale and high sensitivity (77\%) and specificity (81\%) for the Depression subscale [38]. This scale demonstrated acceptable reliability in this study $(\alpha=0.90)$.

Difficulties in Emotion Regulation Scale [DERS: 39]. The DERS is a 36-item, self-report questionnaire that measures overall difficulties in emotion regulation. The DERS consists of six subscales: (1) no acceptance of emotional responses, (2) difficulties engaging in goal-directed behavior, (3) impulse control difficulties, (4) the lack of emotional awareness, (5) limited access, and (6) lack of emotional clarity. Respondents rated their emotional state on 1 (almost never) to 5 (almost always). The total score range of 36-180. A recent study has found that a DERS total score above 97 identified a clinical sample [40]. DERS has high internal consistency $(\alpha=0.93)$. Internal consistency in the current study was acceptable $(\alpha=0.92)$.

\section{Secondary outcomes}

The Positive and Negative Affect Schedule [PANAS: 41]. The PANAS is a brief self-report scale that determines positive and negative affects with two independent ten descriptors. The PANAS demonstrates the two core dimensions of mood positive affect (PA) and negative mood affect (NA). Each item is rated on a five-point scale with a range from very slightly (1) to extremely (5), indicating the extent that the participant has experienced that feeling over the past month. The PANAS has shown highly internally consistent, largely uncorrelated positive affect $(0.89)$ to negative affect $(0.95)$, whereas the discriminant correlations are quite low [41]. Internal consistency in the current study was acceptable $(\alpha=0.80)$.

Penn State Worry Questionnaire [PSWQ: 42]. The PSWQ is a 16-item self-report measure that determines an individual's tendency to worry and intensity and excessiveness of worry on a scale of 1 (not at all typical of me) to 5 (very typical of me). The PSWQ has demonstrated reliable psychometric properties, suitable internal consistency, and test-retest reliability in the local MS population. This measure is suggested for transdiagnostic approach assessments. Internal consistency in the current study was acceptable $(\alpha=0.83)$.

\section{Procedure}

The study was a single-blind, parallel randomized controlled trial comparing psychological intervention group, based on the unified protocol, with a TAU control group. The study, including all assessments and treatments, was conducted at the MS Clinic, located within the MS Centre. The study's methods and procedures were reviewed and approved by the Institutional Human Research 
Ethics Committee and the National Institute for Medical Research and Development, prospectively. "We used the CONSORT checklist and The TIDieR checklist when writing our report (see Additional file 1)."

First, interested PwMS were notified about the study's goals, benefits, and risks, session numbers, randomization, and group allocation chance through telephone or face-to-face interviews. Only who gave verbal informed consent to participate in the study were asked to present their physician agreement or refer to the study participation. The neurologists and clinical psychologist evaluated physician agreements, referrals, medical documents for recent medication prescriptions, and examined the subjects. The eligibility criteria are related to medical conditions obtained; the participants completed the assessment protocol. Individuals who met the SCID-I-IV criteria for depression or anxiety disorders were requested to sign the consent form. All participants obtained a signed written consent form.

At last, only consented subjects who received a valid depression or anxiety diagnosis were selected for randomization. The outcomes were assessed at two timepoints: Time 1: pretreatment to pre-allocation includes baseline, Time 2: immediate after intervention: posttreatment assessment.

\section{Sample size}

The sample size for Analysis of covariance (ANCOVA) was conducted using G*Power 3.1 analysis [43]. A priori power analysis was conducted, using an alpha of 0.05 , a power of 0.8 , and medium to large effect size (Cohen's $f=0.35$ ) to determine the sample size. According to G*Power, the desired total sample size was 64 . Therefore, 70 participants recruited, allowing for a $10 \%$ loss of data (dropping out prior treatment, end treatment assessment).

\section{Randomization and blinding procedures}

Randomization was performed using a computer-generated sequence (www.randomizer.org). A list of anonymous participant identification numbers was used to randomly allocate participants to treatment or control without any restrictions. An independent statistician performed new randomization after each 10-participants allocated. The concealed was disclosed at the end of the study. The independent statistician carried out the randomization and informed the patients and the monitoring board about the allocation. To masking condition assisting, participants were instructed not to disclose any information about the intervention and diagnostic status. Psychological evaluators, data collectors, assessors, and statistic investigators were blinded to the intervention, participants' group, and pervious diagnostic status.

\section{Interventions}

\section{The unified protocol intervention}

The program and sessions were structured based on the latest comprehensive published manual developed by Barlow and colleagues [26-28]. Group therapy consists of 12 weekly 2 -h sessions. The treatment content is included topics about Motivation, psychoeducation, mindfulness, cognitive flexibility, emotion-driven behavior, and emotional avoidance, interoceptive exposure (IE), in vivo exposure, and relapse prevention. The summary of each module content and intervention schedule is demonstrated in Table 2. (See Additional file 2 for the more detailed description.)

\section{Treatment-as-usual intervention}

The control group received the TAU that consists of 12 weekly two hour sessions. The program included psychoeducation and life-long MS considerations (4 sessions), sharing experiences (4 sessions), and marital and parental counseling ( 4 sessions). This treatment could be considered as a psychoeducation intervention

Table 2 Content and the number of sessions for module

\begin{tabular}{lll}
\hline Module & Schedule & Content and the number of sessions for module \\
\hline One & Week 1 & Setting goals and maintaining motivation (1 session) \\
Two & Week 2 & Understanding emotions (1 sessions) \\
Three & Week 3 and $4^{\text {a }}$ & Mindful emotion awareness (2 sessions) \\
Four & Week 5 and 6 & Cognitive flexibility (2 sessions) \\
Five & Week 7 & Countering emotional behaviors (1 sessions) \\
Six & Week 8 & Understanding and confronting physical sensations (1 session) \\
Seven & Weeks 9 to $13^{\text {a }}$ & Emotion exposures (5 sessions) \\
Eight & Week 14 & Recognizing accomplishments and looking to the future (1 session) \\
\hline
\end{tabular}

The Modules of three, four, five, six, and seven are Core modules

a Week 3 and week 13 can be deleted based on the 12 session program 
delivered in routine care focused on reducing negative emotions.

\section{Risk}

Routine medical and psychological evaluations were accomplished before all activities (e.g., assessments, interviews, and treatment sessions). Regarding safety, the medical health care staff included two physicians and four experienced nurses also alerted in case of emergency conditions during all activities. The followup phase coincided with a viral epidemic. Therefore, in order to ensure the safety of participants, no follow-up was performed.

\section{Statistical analysis}

All analyses were conducted with SPSS software version 25 (version 25, SPSS Inc., Chicago, IL), two-tailed with an alpha level of 0.05 to determine statistical significance, following an Intention-to-Treat (ITT) analysis approach. With the ITT approach, study participants are analyzed as members of the treatment group to which they were randomized regardless of their adherence to, or whether they received, the intended treatment. Given that the analysis was based on ITT principles, the data for all randomized 70 individuals were included in the final report. To handle missing data, the last provided data (the last observation-carried-forward (LOCF) were considered as a next point for dropping data. An independent t-test was conducted to explore whether the participant was equivalent at baseline (Time 1).

The parametric test of analysis of covariance (ANCOVA) was conducted to compare the effectiveness of the unified protocol intervention and TAU at post-treatment (Time 2). The scores on the baseline are treated as a covariate to control for pre-existing differences between the groups. Preliminary checks were conducted to ensure no violation of the assumptions of normality, linearity, homogeneity of variances, and homogeneity of regression slopes. Levene's test was used to determine normality and homogeneity of variance. Also, the homogeneity of regression slopes assumption was tested. A paired $t$ test was conducted for all measures between (Time1-Time2) to investigate within groups' changes. The within-group effect size was calculated for both groups.

Effect sizes are reported as partial eta squared. Standardized effect size Cohen's $d$ was calculated for pre-post treatment changes based on means and standard deviations [44]. Effect size estimates were interpreted conservatively, with $0.2,0.5,0.8$, reflecting small, medium, and large treatment effects, respectively.

\section{Results}

\section{Descriptive characteristics at baseline}

There were no significant differences in terms of demographic features, age, duration, education, and other dependent variables at baseline (see Table 1). There was no significant difference between participants with depressive disorders and participants with anxiety disorders in the study $\left(x^{2}=0.91, p=0.33\right)$. The unified protocol participants' ages ranged from 25 to 44 years, with a mean of 33.06 years $(S D=6.22)$. The TAU participants' ages ranged from 22 to 47 years, with a mean of 33.89 years $(S D=5.24)$. Seven $(20 \%)$ from the unified protocol group left the experiment before Time 2. On average, participants had a very high degree of adherence and protocol well tolerated; $28(80 \%)$ of the unified protocol group completed the treatment sessions and completed all the post-treatment measures. Also, 12(37\%) of the TAU group dropped out at post-treatment. Finally, 50 (71\%) of all participants completed the study.

A one-way between groups ANCOVA was conducted to assess the impact of the unified protocol of reported all measures. The ANCOVA assumptions were examined before submitting the test results. Homogeneity of Variance was tested using Levene's test, indicating insignificance of $p$ value $(p>0.05)$. The Homogeneity of Variance assumption was met for: HADS-A $[F(1,68)=3.46, p=.07)$, for HADS$\mathrm{D}[F(1,68)=3.61, p=.06]$ for DERS $F(1,68)=1.89$, $p=.17$, for PANAS-PA $[F(1,68)=1.51, p=.38]$, for PANAS-NA $[F(1,68)=2.80, p=.10]$, and for PSWQ $F(1,68)=1.14, p=.28]$. Also, the homogeneity of regression slopes assumption was met for: HADS-A $[F(1,66)=1.02, p=.32)$, for HADS-D $[F(1$, $66)=1.56, p=.14]$ for DERS $F(1,66)=1.895, p=.17$, for PANAS-PA $[F(1,66)=0.04, p=.84]$, for PANASNA $[F(1,66)=.065, p=.80]$, and for PSWQ $[F(1$, $66)=2.10, p=.15]$. There was no significant interaction between the covariates and the intervention.

\section{Treatment results}

Depression treatment results The ANCOVA was conducted on HADS-D. The results showed a significant main effect for group, $F(1,67)=74.91, p<.001, \eta^{2} p=.52$, and Cohen's $d=1.9$. For the group, the unified protocol significantly less HADS-D scores than TAU. The adjusted post-treatment mean for the unified protocol group $(M=8.01)$ was significantly less than that for the TAU group $(M=12.79)$.

Anxiety treatment results The ANCOVA results showed a significant main effect for group, $F(1,67)=82.47$, $p<.001, \eta^{2} p=.55$, and a Cohen's $d=2.1$. For groups, the unified protocol significantly less HADS-A scores than TAU. The adjusted post-treatment mean for the unified 
protocol group $(M=8.08)$ was significantly less than that for the TAU group $(M=11.68)$.

Emotion dysregulation treatment results The ANCOVA was conducted on DERS. The results showed a significant main effect for group, $F(1,67)=11.04, p=.001, \eta^{2} p=.14$, and a Cohen's $d=0.42$. For the group, the unified protocol significantly lower DERS scores than TAU. The adjusted post-treatment mean for the unified protocol group $(M=97.52)$ was significantly less than that for the TAU group $(M=112.81)$.

Affectivity treatment results The ANCOVA was conducted on PANAS-PA and PANAS-NA. The PANASNA. The results showed a significant main effect for group, $F(1,67)=62.19, p<.001, \eta^{2} p=.48$, and a Cohen's $d=1.89$. The adjusted post-treatment mean for the unified protocol group $(M=18.98)$ was significantly less than that for the TAU group $(M=27.50)$.

The PANAS-PA results showed a significant main effect for group, $F(1,67)=47.99 p<.001, \eta^{2} p=.42$, and a Cohen's $d=1.51$. The adjusted post-treatment mean for the unified protocol group $(M=30.68$. $)$ was significantly higher than that for the TAU group $(M=24.83)$.

Worry treatment results The ANCOVA was conducted on PSWQ. The results showed a significant main effect for group, $F(1,67)=34.78, p<.001, \eta^{2} p=.34$, and a Cohen's $d=1.2$. The adjusted post-treatment mean for the unified protocol group $(M=34.24)$ was significantly lower than that for the TAU group $(M=46.26)$.

A Paired $t$ test was carried out to examine treatment effectiveness between Time 2 and Time1. These findings revealed the unified protocol had a significant effect on symptom improvement (see Table 3). Means and standard deviations were calculated at Time1 and Time 2 (see

Table 3 Paired $t$ test and within group effect size at postintervention

\begin{tabular}{|c|c|c|c|c|c|}
\hline \multirow[t]{2}{*}{ Item } & \multicolumn{2}{|c|}{$\begin{array}{l}\text { Treatment } \\
\text { as usual } \\
\text { group }\end{array}$} & \multicolumn{3}{|c|}{ Unified protocol group } \\
\hline & $t(34)$ & $p$ & $t(34)$ & $p$ & Cohens'd $[95 \% \mathrm{Cl}]$ \\
\hline Anxiety & 1.95 & .062 & 10.54 & $p<.001$ & $\begin{array}{c}-2.78[-3.67 \\
-2.01]\end{array}$ \\
\hline Depression & -.46 & .643 & 7.83 & $p<.001$ & $\begin{array}{l}-2.01[-2.78 \\
-1.35]\end{array}$ \\
\hline $\begin{array}{l}\text { Emotion difficul- } \\
\text { ties }\end{array}$ & -2.01 & .052 & 3.07 & $p<.01$ & $\begin{array}{l}-0.20[-0.66 \\
-0.14]\end{array}$ \\
\hline Positive affect & 1.98 & .055 & -7.01 & $p<.001$ & $1.44[0.86,2.08]$ \\
\hline Negative affect & -.98 & .327 & 8.26 & $p<.001$ & $\begin{array}{l}-1.95[-2.65 \\
-1.31]\end{array}$ \\
\hline Worry & .65 & .512 & 6.89 & $p<.001$ & $\begin{array}{l}-1.45[-2.02 \\
-0.92]\end{array}$ \\
\hline
\end{tabular}

Time2-Time1 post-treatment to baseline, $\mathrm{Cl}$ confidence interval of the difference
Table 4). There were no adverse events associated with this trial. A comparison test Between Time 2 and Time 1 revealed no significant differences for the TAU group.

The SCID-I -IV demonstrated 21 of 35 participants in the unified protocol group (60\%) no longer met the diagnostic criteria for their principal diagnosis at the end of the study. The SCID-I-IV demonstrated no worse condition for all participants at Time2.

\section{Discussion}

MS is associated with a broad array of emotional disorders, negative symptoms, social interference, and physical disability that compromise well-being [45]. This study aimed to examine the efficacy of a group format of the unified protocol for the transdiagnostic treatment of emotional disorders and symptoms in adult PwMS. Our approach was based on the key development of the emotion regulation mechanism outlined in the unified protocol transdiagnostic treatment framework for emotional disorders. The results revealed that PwMS, who participated in the unified protocol intervention group, demonstrated significant improvements in depressive and anxiety and worry emotion regulation, and affectivity outcomes at post-treatment compared with those who participated in the TAU group. Our findings revealed significant changes in depression measure, in anxiety measure, and in worry, in emotion regulation measure, and affectivity measure in the unified protocol group at posttreatment relative to baseline.

The results are consistent with studies that indicate the unified protocol is effective in improving emotional disorders [46-48]. The core modules of the unified protocol are relevant to depression. Briefly, negative affect (e.g., sadness, shame, anger) and maladaptive, avoidant reactions to negative affect are targeted in the unified protocol. For example, in emotional disorders, worrying is a critical maladaptive cognitive process contributing

\begin{tabular}{|c|c|c|c|c|}
\hline \multirow[t]{2}{*}{ Item } & \multicolumn{2}{|c|}{ Treatment as usual group } & \multicolumn{2}{|c|}{ Unified protocol group } \\
\hline & Time1 & Time2 & Time1 & Time2 \\
\hline Anxiety & $12.40(1.37)$ & $11.69(1.65)$ & $12.57(1.40)$ & $8.09(1.82)$ \\
\hline Depression & $12.60(1.65)$ & $12.80(2.00)$ & $12.88(1.80)$ & $7.9(2.79)$ \\
\hline $\begin{array}{l}\text { Emotion dif- } \\
\text { ficulties }\end{array}$ & $108.74(8.46)$ & $114.3(16.63)$ & $110.1(12.17)$ & $106.5(20.42)$ \\
\hline Positive affect & $26.43(3.98)$ & $25.00(3.40)$ & $25.20(3.36)$ & $30.51(3.79)$ \\
\hline $\begin{array}{l}\text { Negative } \\
\text { affect }\end{array}$ & $26.69(3.03)$ & $27.51(3.77)$ & $27.43(3.27)$ & $18.97(5.04)$ \\
\hline Worry & $47.00(8.42)$ & $46.09(8.60)$ & $48.00(8.86)$ & $34.43(9.40)$ \\
\hline
\end{tabular}

Time1: pre-treatment, Time2: post-treatment to 
to the maintenance of the disorder, and worrying can be effectively targeted by promoting adaptive emotion regulation strategies [49]. PwMS focus on the disease consequences, which may be concluded to catastrophizing future, over-estimate threat, and under-estimate their abilities to cope. Present-Focused Emotion, a core module in the unified protocol, helps the patients recognize their thoughts and feelings, and concentrate on the current condition demands, making emotional experiences feel more under control and manageable. The improvement of emotion regulation can be associated with an improvement in depression and anxiety symptoms [50].

Findings revealed significant changes in DERS at posttreatment regarding with TAU group. This study develops the unified protocol benefits on difficulties emotion regulation scale have improved other clinical outcomes [51]. Also, the results provide supports for the application of emotion regulation in promoting adaptive emotion regulation among people with mental disorders [52]. In line with our investigation, numerous researches have replicated the emotional regulation implication in the treatment of depression [53], anxiety disorders [54], excessive worry, and psychological stress [21].

A large Cohen's $d$ in the negative and positive affect was found with a higher significant effect on negative affect than positive affect. These results are the same that previous RCT, applying unified protocol in emotional disorders samples that have found changes in neuroticism/negative affect after unified protocol intervention [55]. Some studies have also found differences in extraversion/positive affect [56]. The reduction in neuroticism scores confirms the unified protocol's theory, an emotion regulation intervention targeting specifically neuroticism/negative affect [20], a psychopathology mechanism associated with the etiology of the emotional disorders [57]. These results suggest unified protocol, which typically focuses on reductions in negative affect, may also improve positive affect. Positive affect is a malleable construct and can be influenced by unified protocol. Change in positive affect can be potentially associated with improved both psychological and clinical outcomes [58]. One possible reason could be that unified protocol helps patients understand the relevant function of all range of emotions, including "positive emotions" such as happiness, joy, pride. Another possibility could be related to the group format delivery. Group therapy facilitates the normalization of MS-related experiences, and sharing with similar people reduces the stigma associated with psychological treatment.

The current study could develop the unified protocol as a transdiagnostic approach, consisting of five core modules and practical techniques for addressing different aspects of emotion regulation. Emotion dysregulation predicts quality of life, independently of disease severity and cognitive functioning [59]. Moreover, Emotional distress associated with maladaptive coping strategies is led to poor well-being rather than disease duration or severity [60]. For example, Emotional problems among mothers with MS negatively associate with the mother's ability to cope with the disease and positively associate with depressive symptoms in their healthy partners [61]. The unified protocol components, such as Psychoeducational courses, emotional skills, and stress self-management techniques can be beneficial to enhance well-being in MS [62]. Awareness of thoughts, beliefs, and interactions facilitates coping in PwMS [63]. Interoceptive exposure is another component of the unified protocol. PwMS are more sensitive to visceral sensations than healthy individuals [64]. Dysfunctions in interoceptive inference could underlie a range of pathologies such as anxiety [65]. In PwMS, bodily sensations are usually associated with high anxiety. Interoceptive exposure may be beneficial and facilitate a controlled coping behavior, and less stress react, gradually [66]. Also, individuals with better interoceptive perception demonstrate greater self-regulatory ability in experimental social interaction [67]. Meta-analytic evidence supports the use of the mindfulness-based intervention in PwMS to improve fatigue [68].

The study revealed surprising findings. There was no significant difference between the participants who received a depressive disorder diagnosis and those who received an anxiety disorder diagnosis at baseline. This finding is contrary to current insight and epidemiologic data in PwMS [69]. This finding is critical because anxiety receives far less attention in MS. DERS scores are related to both depression and anxiety levels in the MS sample [59]. According to DERS mean score at baseline, difficulties with emotion regulation is very high in PwMS.

We investigated the unified protocol's feasibility in a group format to an MS transdiagnostic sample with emotion regulation problems. According to evidence considering that emotion dysregulation is connected with less willingness to participate in psychological trials [70], we classified the sample as challenging to treat. According to the treatment retention rate in this study $(71 \%)$, the treatment was well tolerated. Also, the results are in line with the data provided previous trials $[29,71]$ confirm a significant improvement of patients treated in a group format.

\section{Limitations}

The results from this trial should be interpreted in the context of several limitations. First, the participants were generally well-educated, which can be enhanced their abilities to gain more the unified protocol and diminish the results' generalizability. A priori power analysis was conducted, using an alpha of 0.05. However, for three 
primary outcomes variables (HADS-A, HADS-D, and DERS) an alpha of 0.0167 should have been considered. The next limitation was that the no-follow-up made it difficult to assess prevention effects. One strength point of this study was the SCID-I-IV application at enrollment and post-treatment.

\section{Conclusion}

Overall the findings provide the support that the unified protocol could be an additional efficient as a parsimonious, transdiagnostic treatment of emotional disorders for adult PwMS. Although the results are promising, more research should be conducted to extend the findings obtained in this study. Transdiagnostic research has the potential to represent better the clinical and scientific reality of mental health problems, reflecting the complexity and comorbidity that is the norm in clinical practice. The unified protocol is equally effective as goldstandard specific disorder protocols in people without MS. Future trials are required to investigate the unified protocol effectiveness, compared with gold-standard specific disorder protocols in the PwMS. Further studies are required to assess the cost-effectiveness and efficacy of the unified protocol intervention with larger samples to promote it as part of routine care for PwMS. Economic evaluations can be simultaneously incorporated in future trials, as this has not yet been formally evaluated. There would be value in adding qualitative components into future trials to establish the unified protocol interventions' acceptability for both clinicians and clients. The findings developed the unified protocol as a mechanistically transdiagnostic approach might be applied across multiple disorders. In a unified approach, thoughts, behaviors, feelings, and body sensations are interacting dynamically, and emotional experiences influence each of them. Unified protocol Transdiagnostic intervention facilitates PwMS to learn how to respond to their unpleasant emotions more adaptively. Given the negative consequences of anxiety and depression in PwMS, interventions such as this may reduce the risk of these adverse outcomes and produce benefits for PwMS.

\section{Supplementary information}

Supplementary information accompanies this paper at https://doi. org/10.1186/s40359-020-00480-8.

Additional file 1. Consort.

Additional file 2. Treatment Protocol.

\section{Abbreviations}

ANCOVA: Analysis of covariance; CBT: Cognitive Behavioral Therapy; DERS: Difficulties in Emotion Regulation Scale; DSM: Diagnostic and Statistical Manual of Mental Disorders; HADS: The hospital anxiety and depression scale; ITT: Intention-to-Treat; LOCF: Last observation-carried-forward; MS: Multiple sclerosis; PANAS: Positive and negative affect scale; PSWQ: Penn State Worry Questionnaire; PWMS: People with MS; SCID I-IV: Structured Clinical Interview for DSM-IV Axis I Disorders; TAU: Treatment-as-usual.

\section{Acknowledgements}

Not applicable.

Authors' contributions

NN and MS designed the study protocol with important contributions from EG Translated the original unified protocol manuals. NN and DM and all their collaborators are in charge of recruiting study participants. All authors contributed in drafted the manuscript. All authors participated in the review and revision of the manuscript and have approved the final manuscript to be published. EG and DM were the study methodologist. EG also contributed to the revision. All authors read and approved the final manuscript.

\section{Funding}

The authors received no financial support for the research, authorship, and/or publication of this article.

\section{Availability of data and materials}

The data that support the findings of this study are available on request from the corresponding author.

\section{Ethics approval and consent to participate}

The study has been performed in accordance with the Declaration of Helsinki and has been approved and registered by the ethical and research committees from the following collaborating centers. The study, including all assessments and procedures for the study, was reviewed by the National Institute for Medical Research and Development (NIMAD); prospectively (number: IRCT20190711044173N1; Registered at 31octobe2019, https://en.irct.ir/user/ trial/40779/view). All participants provided a signed written consent.

\section{Consent for publication}

Not applicable.

\section{Competing interests}

The authors declare no potential conflicts of interest with respect to the research, authorship, and/or publication of this article.

Received: 20 April 2020 Accepted: 22 October 2020

Published online: 31 October 2020

\section{References}

1. Feinstein A, Brochet B, Sumowski J. The cognitive effects of anxiety and depression in immune-mediated in flammatory diseases. Neurology. 2019. https://doi.org/10.1212/WNL.0000000000006840.

2. Grech LB, Butler E, Stuckey S, Hester R. Neuroprotective benefits of antidepressants in multiple sclerosis: are we missing the mark? J Neuropsychiatry Clin Neurosci. 2019;31(4):289-97. https://doi.org/10.1176/appi. neuropsych.18070164.

3. Marrie RA, Patten SB, Berrigan LI, et al. Diagnoses of depression and anxiety versus current symptoms and quality of life in multiple sclerosis. Int J MS Care. 2018;20(2):76-84. https://doi.org/10.7224/1537-2073.2016-110.

4. Corallo F, Bonanno L, Di Cara M, et al. Therapeutic adherence and coping strategies in patients with multiple sclerosis. Medicine. 2019;98(29):e1. https://doi.org/10.1097/md.0000000000016532.

5. McKay KA, Tremlett H, Fisk JD, et al. Psychiatric comorbidity is associated with disability progression in multiple sclerosis. Neurology. 2018;90(15):e1316-1323. https://doi.org/10.1212/wnl.0000000000005302.

6. Bronnum-Hansen $\mathrm{H}$. Suicide among Danes with multiple sclerosis. J Neurol Neurosurg Psychiatry. 2005;76(10):1457-9. https://doi.org/10.1136/ jnnp.2004.056747.

7. Feinstein A, Pavisian B. Multiple sclerosis and suicide. Mult Scler J. 2017;23(7):923-7. https://doi.org/10.1177/1352458517702553.

8. Hawkes $\mathrm{CH}$, Boniface D. Risk associated behavior in premorbid multiple sclerosis: a case-control study. Mult Scler Relat Disord. 2014;3(1):40-7. https://doi.org/10.1016/j.msard.2013.05.002. 
9. Skokou M, Soubasi E, Gourzis P. Depression in multiple sclerosis: a review of assessment and treatment approaches in adult and pediatric populations. ISRN Neurol. 2012;2012:1-6. https://doi.org/10.5402/2012/427102.

10. Strober LB, Arnett PA. Depression in multiple sclerosis: the utility of common self-report instruments and development of a diseasespecific measure. J Clin Exp Neuropsychol. 2015;37:722-32. https://doi. org/10.1080/13803395.2015.1063591.

11. Newby JM, McKinnon A, Kuyken W, Gilbody S, Dalgleish T. Systematic review and meta-analysis of transdiagnostic psychological treatments for anxiety and depressive disorders in adulthood. Clin Psychol Rev. 2015;40:91-110. https://doi.org/10.1016/j.cpr.2015.06.002.

12. Holmes EA, O'Connor RC, Perry VH, et al. Multidisciplinary research priorities for the COVID-19 pandemic: a call for action for mental health science. Lancet Psychiatry. 2020. https://doi.org/10.1016/S2215 -0366(20)30168-1.

13. Fiest KM, Walker JR, Bernstein CN, et al. Systematic review and metaanalysis of interventions for depression and anxiety in persons with multiple sclerosis. Mult Scler Relat Disord. 2016;5(3):12-26. https://doi. org/10.1016/j.msard.2015.10.004.

14. Simpson R, Mair FS, Mercer SW. Mindfulness-based stress reduction for people with multiple sclerosis - a feasibility randomised controlled trial. BMC Neurol. 2017;17(1):1-12. https://doi.org/10.1186/s12883-017-0880-8.

15. Sesel AL, Sharpe L, Naismith SL. Efficacy of psychosocial interventions for people with multiple sclerosis: a meta-analysis of specific treatment effects. Psychother Psychosom. 2018;87(2):105-11. https://doi. org/10.1159/000486806.

16. Clark LA, Cuthbert B, Lewis-Fernández R, Narrow WE, Reed GM. Three approaches to understanding and classifying mental disorder: ICD-11, DSM-5, and the National Institute of Mental Health's Research Domain Criteria (RDoC). Psychol Sci Public Interest. 2017;18(2):72-145. https://doi. org/10.1177/1529100617727266.

17. Norton PJ, Paulus DJ. Toward a unified treatment for emotional disorders: update on the science and practice. Behav Ther. 2016;47(6):854-68. https ://doi.org/10.1016/j.beth.2015.07.002.

18. Butler E, Matcham F, Chalder T. A systematic review of anxiety amongst people with Multiple Sclerosis. Mult Scler Relat Disord. 2016;10:145-68. https://doi.org/10.1016/j.msard.2016.10.003.

19. Sauer-Zavala S, Gutner CA, Farchione TJ, Boettcher HT, Bullis JR, Barlow DH. Current definitions of "transdiagnostic" in treatment development: a search for consensus. Behav Ther. 2017;48(1):128-38. https://doi. org/10.1016/j.beth.2016.09.004.

20. Brown TABD. A proposal for a dimensional classification system based on the shared features of the DSM-IV anxiety and mood disorders: implications for assessment and treatment. Psychol Assess. 2009;21(3):256. https ://doi.org/10.1037/a0016608.

21. MacNamara A, Kotov R, Hajcak G. Diagnostic and symptom-based predictors of emotional processing in generalized anxiety disorder and major depressive disorder: an event-related potential study. Cognit Ther Res. 2015;40(3):275-89. https://doi.org/10.1007/s10608-015-9717-1.

22. Carrigan N, Dysch L, Salkovskis P. The impact of health anxiety in multiple sclerosis: a replication and treatment case series. Behav Cogn Psychother. 2018;46(2):148-67. https://doi.org/10.1017/S135246581700056X.

23. Ellard KK, Barlow DH, Whitfield-Gabrieli S, Gabrieli JDE, Deckersbach T. Neural correlates of emotion acceptancevsworry or suppression in generalized anxiety disorder. Soc Cogn Affect Neurosci. 2017;12(6):1009-21. https://doi.org/10.1093/scan/nsx025.

24. Nazari N. The effectiveness of hope therapy on improving marital adaptation in women with multiple sclerosis (MS). J Adv Pharm Educ Res. 2020;10(S1):49-54.

25. Houtchens MK, Dessa Sadovnick A. Health issues in women with multiple sclerosis. Vienna: Springer; 2017. https://doi. org/10.1007/978-3-7091-4858-7.

26. Barlow DH. Unified protocol for transdiagnostic treatment of emotional disorders. Therapist guide therapist guide. Oxford: Oxford University Press; 2011.

27. Barlow DH, Farchione TJ, Bullis JR, et al. The unified protocol for transdiagnostic treatment of Emotional Disorders compared with diagnosisspecific protocols for anxiety disorders: a randomized clinical trial. JAMA Psychiatry. 2017;74(9):875-84. https://doi.org/10.1001/jamapsychi atry.2017.2164.
28. Barlow DH. Applications of the unified protocol for transdiagnostic treatment of emotional disorders. New York: Oxford University Press; 2018.

29. Osma J, Suso-Ribera C, García-Palacios A, et al. Efficacy of the unified protocol for the treatment of emotional disorders in the Spanish public mental health system using a group format: study protocol for a multicenter, randomized, non-inferiority controlled trial. Health Qual Life Outcomes. 2018;16(1):46. https://doi.org/10.1186/s12955-018-0866-2.

30. Sakiris N, Berle D. A systematic review and meta-analysis of the Unified Protocol as a transdiagnostic emotion regulation based intervention. Clin Psychol Rev. 2019;72:101751. https://doi.org/10.1016/j.cpr.2019.101751.

31. Allen $L B$, Tsao JCl, Seidman LC, Program PP, Geffen D. A unified, transdiagnostic treatment for adolescents with chronic pain and comorbid anxiety and depression. Cogn Behav Pract. 2012;19(1):56-67. https://doi. org/10.1016/j.cbpra.2011.04.007.

32. Bullis JR, Sauer-Zavala S, Bentley KH, Thompson-Hollands J, Carl JR, Barlow $\mathrm{DH}$. The unified protocol for transdiagnostic treatment of emotional disorders. Behav Modif. 2015;39:295-321. https://doi.org/10.1177/01454 45514553094.

33. American Psychiatric Association. Diagnostic and Statistical Manual of Mental Disorders DSM-IV-TR (Text Revision) Details: American Psychiatric Association. Diagnostic Stat Man Ment Disord DSM-IV-TR. 2000:282.

34. First MB, Department NYSPIBR. Structured clinical interview for DSM-IV axis I disorders: SCID-I. New York: American Psychiatric Press; 1997.

35. First MB, Spitzer RL, Gibbon M, Williams JB. Structured clinical interview for DSM-IV-TR axis I disorders. New York: New York State Psychiatric Institute; 2002.

36. Sharifi V, Assadi SM, Mohammadi MR, et al. A Persian translation of the structured clinical interview for diagnostic and statistical manual of mental disorders, Fourth Edition: psychometric properties. Compr Psychiatry. 2009;50(1):86-91. https://doi.org/10.1016/j.comppsych.2008.04.004.

37. Zigmond AS, Snaith RP. The hospital anxiety and depression scale. Acta Psychiatr Scand. 1983;67(6):361-70. https://doi. org/10.1111/j.1600-0447.1983.tb09716.x.

38. Watson TM, Ford E, Worthington E, Lincoln NB. Validation of mood measures for people with multiple sclerosis. Int J MS Care. 2014;16(2):105-9. https://doi.org/10.7224/1537-2073.2013-013.

39. Gratz KL, Roemer L. Multidimensional assessment of emotion regulation and dysregulation: development, factor structure, and initial validation of the difficulties in emotion regulation scale. J Psychopathol Behav Assess. 2004;26(1):41-54. https://doi.org/10.1023/B:JOBA.0000007455.08539.94.

40. Neacsiu AD, Eberle JW, Kramer R, Wiesmann T, Linehan MM. Dialectical behavior therapy skills for transdiagnostic emotion dysregulation: a pilot randomized controlled trial. Behav Res Ther. 2014;59:40-51. https://doi. org/10.1016/j.brat.2014.05.005.

41. Tellegen A, Watson D, Clark LA. Development and validation of brief measures of positive and negative affect: the PANAS scales. J Pers Soc Psychol. 1988;54(6):1063-70.

42. Meyer TJ, Miller ML, Metzger RL, Borkovec TD. Penn State Worry Questionnaire. 1990;1:9-10. https://doi.org/10.1037/t01760-000

43. Faul F, Erdfelder E, Lang A-G, Buchner A. Statistical power analyses using G*Power 3.1: tests for correlation and regression analyses. Behav Res Methods. 2009;41(4):1149.

44. Kline RB. Becoming a behavioral science researcher. 2nd ed. New York: Guilford Press; 2020.

45. Salhofer-Polanyi S, Friedrich F, Löffler S, et al. Health-related quality of life in multiple sclerosis: temperament outweighs EDSS. BMC Psychiatry. 2018;18(1):143. https://doi.org/10.1186/s12888-018-1719-6.

46. Sauer-Zavala S, Bentley KH, Steele SJ, et al. Treating depressive disorders with the Unified Protocol: a preliminary randomized evaluation. J Affect Disord. 2020;264:438-45. https://doi.org/10.1016/j.jad.2019.11.072.

47. Boswell JF, Anderson LM, Barlow DH. An idiographic analysis of change processes in the unified transdiagnostic treatment of depression. J Consult Clin Psychol. 2014;82(6):1060-71. https://doi.org/10.1037/a0037403.

48. Bentley KH. Applying the unified protocol transdiagnostic treatment to nonsuicidal self-injury and co-occurring emotional disorders: a case illustration. J Clin Psychol. 2017;73(5):547-58. https://doi.org/10.1002/ jclp.22452.

49. Hofmann SG, Sawyer AT, Fang A, Asnaani A. Emotion dysregulation model of mood and anxiety disorders. Depress Anxiety. 2012;29(5):40916. https://doi.org/10.1002/da.21888. 
50. Sloan E, Hall K, Moulding R, Bryce S, Mildred H, Staiger PK. Emotion regulation as a transdiagnostic treatment construct across anxiety, depression, substance, eating and borderline personality disorders: a systematic review. Clin Psychol Rev. 2017;57:141-63. https://doi.org/10.1016/j. cpr.2017.09.002.

51. Mohajerin B, Bakhtiyar M, Olesnycky OS, Dolatshahi B, Motabi F. Application of a transdiagnostic treatment for emotional disorders to body dysmorphic disorder: a randomized controlled trial. J Affect Disord. 2018;2019(245):637-44. https://doi.org/10.1016/j.jad.2018.11.058.

52. Gratz KL, Weiss NH, Tull MT. Examining emotion regulation as an outcome, mechanism, or target of psychological treatments. Curr Opin Psychol. 2015;3:85-90.

53. Marroquín B, Nolen-Hoeksema S. Emotion regulation and depressive symptoms: close relationships as social context and influence. J Pers Soc Psychol. 2015;109(5):836-55. https://doi.org/10.1037/pspi0000034.

54. Klemanski DH, Curtiss J, McLaughlin KA, Nolen-Hoeksema S. Emotion regulation and the transdiagnostic role of repetitive negative thinking in adolescents with social anxiety and depression. Cogn Ther Res. 2016;41(2):206-19. https://doi.org/10.1007/s10608-016-9817-6.

55. Laposa JM, Mancuso E, Abraham G, Loli-Dano L. Unified protocol transdiagnostic treatment in group format. Behav Modif. 2016;41:253-68.

56. Farchione TJ, Fairholme CP, Ellard KK, et al. Unified protocol for transdiagnostic treatment of emotional disorders: a randomized controlled trial. Behav Ther. 2012:43:666-78.

57. Norton PJ, Paulus DJ. Transdiagnostic models of anxiety disorder: theoretical and empirical underpinnings. Clin Psychol Rev. 2017;56:122-37. https://doi.org/10.1016/j.cpr.2017.03.004.

58. Wilner Tirpak J, Cassiello-Robbins C, Ametaj A, et al. Changes in positive affect in cognitive-behavioral treatment of anxiety disorders. Gen Hosp Psychiatry. 2019;61:111-5. https://doi.org/10.1016/j.genhosppsy ch.2019.06.008

59. Phillips LH, Henry JD, Nouzova E, Cooper C, Radlak B, Summers F. Difficulties with emotion regulation in multiple sclerosis: links to executive function, mood, and quality of life. J Clin Exp Neuropsychol. 2014;36(8):83142. https://doi.org/10.1080/13803395.2014.946891.

60. Harrison AM, McCracken LM, Bogosian A, Moss-Morris R. Towards a better understanding of MS pain: a systematic review of potentially modifiable psychosocial factors. J Psychosom Res. 2015;78(1):12-24. https://doi. org/10.1016/j.jpsychores.2014.07.008.

61. Steck B, Amsler F, Kappos L, Bürgin D. Gender-specific differences in the process of coping in families with a parent affected by a chronic somatic disease (e.g. Multiple Sclerosis). Psychopathology. 2001;34:236-44.
62. Kidd T, Carey N, Mold F, et al. A systematic review of the effectiveness of self-management interventions in people with multiple sclerosis at improving depression, anxiety and quality of life. PLoS ONE. 2017;12(10):e0185931. https://doi.org/10.1371/journal.pone.0185931.

63. Lincoln NB, Yuill F, Holmes J, et al. Evaluation of an adjustment group for people with multiple sclerosis and low mood: a randomized controlled trial. Mult Scler J. 2011;17:1250-7.

64. Kahraman T, Özdoğar AT, Ertekin Ö, Özakbaş S. Frequency, type, distribution of pain and related factors in persons with multiple sclerosis. Mult Scler Relat Disord. 2018;2019(28):221-5. https://doi.org/10.1016/j.msard .2019.01.002.

65. Seth AK. Interoceptive inference, emotion, and the embodied self. Trends Cogn Sci. 2013;17(11):565-73. https://doi.org/10.1016/j.tics.2013.09.007.

66. Oser M, Khan A, Kolodziej M, Gruner G, Barsky AJ, Epstein L. Mindfulness and interoceptive exposure therapy for anxiety sensitivity in atrial fibrillation: a pilot study. Behav Modif. 2019. https://doi.org/10.1177/01454 45519877619.

67. Ferri F, Chiarelli AM, Merla A, Gallese V, Costantini M. The body beyond the body: expectation of a sensory event is enough to induce ownership over a fake hand. Proc R Soc B Biol Sci. 2013;280(1765):20131140. https:// doi.org/10.5061/dryad.8f251.

68. Simpson R, Simpson S, Ramparsad N, Lawrence M, Booth J, Mercer SW. Effects of mindfulness-based interventions on physical symptoms in people with multiple sclerosis - a systematic review and meta-analysis. Mult Scler Relat Disord. 2020;38:101493. https://doi.org/10.1016/j.msard 2019.101493.

69. Marrie RA, Fisk JD, Tremlett H, et al. Differences in the burden of psychiatric comorbidity in MS vs the general population. Neurology. 2015;85(22):1972-9. https://doi.org/10.1212/wnl.0000000000002174.

70. Vogel DL, Wade NG, Hackler AH. Emotional expression and the decision to seek therapy: the mediating roles of the anticipated benefits and risks. J Soc Clin Psychol. 2008;27(3):254-78. https://doi.org/10.1521/ jscp.2008.27.3.254.

71. Reinholt N, Aharoni R, Winding C, Rosenberg N, Rosenbaum B, Arnfred S. Transdiagnostic group CBT for anxiety disorders: the unified protocol in mental health services. Cogn Behav Ther. 2017;46(1):29-43. https://doi. org/10.1080/16506073.2016.1227360.

\section{Publisher's Note}

Springer Nature remains neutral with regard to jurisdictional claims in published maps and institutional affiliations.
Ready to submit your research? Choose BMC and benefit from:

- fast, convenient online submission

- thorough peer review by experienced researchers in your field

- rapid publication on acceptance

- support for research data, including large and complex data types

- gold Open Access which fosters wider collaboration and increased citations

- maximum visibility for your research: over $100 \mathrm{M}$ website views per year

At BMC, research is always in progress.

Learn more biomedcentral.com/submissions 